\title{
THE EFFECT OF PULSE RATE ON VACUUM PHOTOTRIODES RESPONSE AND THE USE OF AN LED PULSER TO IMPROVE STABILITY
}

\author{
DAWN E. LESLIE*+ \\ School of Engineering and Design, Brunel University, Uxbridge UB8 3PH, UK
}

\begin{abstract}
The Endcap Electromagnetic Calorimeter of the Compact Muon Solenoid detector (CMS) at the Large Hadron Collider (LHC) uses vacuum phototriodes (VPTs), which operate in the full 3.8T magnetic field of the experiment, to detect the scintillation light from the lead tungstate crystals. Initial measurements of the variation in response of VPTs, induced by sudden changes in the illuminating light pulse rate, prompted the inclusion of a dedicated stability pulser based on light emitting diodes (LEDs). The response of production VPTs, under simulated LHC operating conditions, has been investigated in three independent studies: in-situ tests with the installed endcaps at CERN, and separate VPT studies by groups at the University of Virginia, USA and Brunel University, UK. In this work, results are presented which illustrate the magnitude of the effect to demonstrate the expected stability of the VPTs during normal LHC operation, with a proposed regime for operating the stability pulser to minimise variations in response. It is demonstrated that a continuous signal at a rate of $100 \mathrm{~Hz}$ is sufficient to reduce the change in the VPT response to $<0.2 \%$.
\end{abstract}

\section{Introduction}

$\mathrm{CMS}^{1}$ is one of two general purpose particle physics experiments recently installed at the Large Hadron Collider (LHC) at CERN. The electromagnetic calorimeter uses large monocrystals of the scintillator lead tungstate coupled to sensitive photodetectors. The development of small radiation tolerant vacuum phototriodes (VPTs) which detect the scintillation light in the endcap region of the electromagnetic calorimeter (EE), has been previously reported ${ }^{2}$. Since then, 16100 production VPTs (type PMT188) have been manufactured to CMS specifications by Research Institute Electron (RIE), St Petersburg, Russia ${ }^{3}$ and 14648 were installed in the endcaps.

The VPTs used in CMS are single gain-stage devices with a diameter of $26 \mathrm{~mm}$ and an active area of approximately $280 \mathrm{~mm}^{2}$. In the absence of electrostatic focussing, VPTs require the presence of a strong, quasi-axial magnetic field for stable operation and as such, the devices comprise an anode of very fine $(10 \mu \mathrm{m}$ pitch) copper mesh, allowing them to operate in the $3.8 \mathrm{~T}$ field.

VPTs have been seen to exhibit changes in response which are related to the average current being drawn from them ${ }^{1}$. To minimise these effects during repeated LHC beam-on and beam-off cycles a dedicated LED pulser system, comprising blue and orange LEDs, has been installed in the endcaps.

\footnotetext{
* Corresponding author: Tel. +44 1895 267367; Fax. +44 1895 269805; email. dawn.leslie@ brunel.ac.uk

+ On behalf of the CMS ECAL Group
} 


\section{In situ and Laboratory Tests}

\subsection{Data from VPT in the CMS endcaps}

The response of the production VPTs has been evaluated during a month-long data taking exercise in late 2008 (Cosmic Run at Four Tesla (CRAFT)), by supplying a pulsed load light to simulate a typical cycle of LHC operation. Initial in-situ tests of 200 VPTs showed an average $0.4 \%$ decrease in response at $\mathbf{B}=0 \mathrm{~T}$ during the application of the load of $10 \mathrm{kHz}$, followed by a $4-5 \%$ change in response when the load was removed, as shown in Figure $1^{4}$. By contrast, with the solenoid operating at its nominal axial magnetic field of $3.8 \mathrm{~T}$, the average response of the VPTs increased during application of the load by $\sim 0.4 \%$ and decreased by $0.1 \%$ when the load was removed. Tests are underway to see if the residual effects at $3.8 \mathrm{~T}$ are reduced in the presence of a constant low-rate background signal.

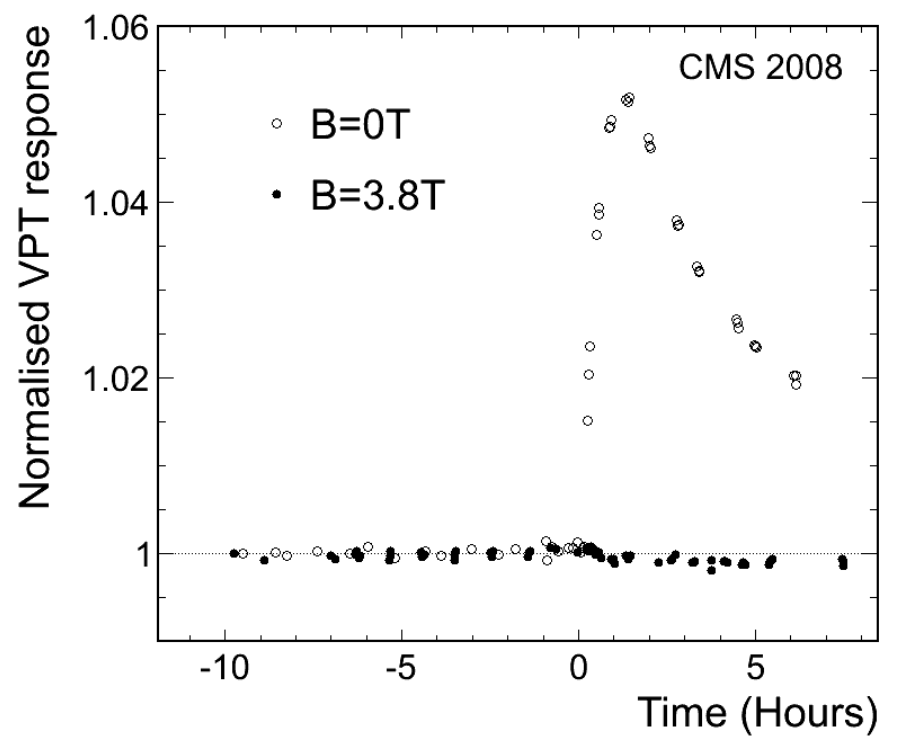

Figure 1. Average normalised response of 200 VPTs for two high rate LED pulsing tests at $\mathbf{B}=0 \mathrm{~T}$ (open circles) and $\mathbf{B}=3.8 \mathrm{~T}$ (filled circles) at CRAFT. In both tests, LED pulsing with a rate of $10 \mathrm{kHz}$ was performed for a period of 17 hours and turned off at the point $\mathrm{T}=0$ hours. The VPT response was normalised to the value at $\mathrm{T}=-10$ hours in both tests. 


\subsection{Extended tests on an individual VPT}

Recent tests, conducted at Brunel University, have concentrated on one production VPT operated continuously at $15^{\circ}$ to a $4.0 \mathrm{~T}$ field over an extended period of time, currently in excess of six months. Tests have been carried out during a simulated LHC cycle i.e. with a constant signal of $100 \mathrm{~Hz}$ during both the 'off' period of 8 hours (latterly 4 hours) and the 'on' period of 16 hours ( 20 hours), when a load pulse is added to simulate the average current that would be present in the endcaps at nominal LHC luminosity. Figure 2 shows the most recent results of VPT response as a function of charge taken from the photocathode of the VPT. The experimental arrangement at Brunel uses two separate LEDs, both temperature stabilised, to supply the signal and load pulses and a pin diode provides a reference. The stability of the VPT was studied by exposing the photocathode to blue LED light - black data points correspond to a cathode current of $0.25 \mathrm{nA}$, with the grey data points resulting from an increased cathode current of $1 \mathrm{nA}$ (although from $6.0 \times 10^{-3} \mathrm{C}$ onwards, the signal rate was reduced to $10 \mathrm{~Hz}$ ). $1 \mathrm{nA}$ is the predicted cathode photocurrent drawn at $\eta=2.1$ at $\mathrm{L}=10^{34} \mathrm{~cm}^{-2} \mathrm{~s}^{-1}$. It should be noted that the $\mathrm{x}$-axis zero is a relative zero, as the VPT under investigation had been used in tests prior to this study, providing an initial cathode charge of $\sim 0.6 \mathrm{mC}$. The y-axis shows the ratio of the peak of the VPT signal to that of the pin reference photodiode monitoring the LED intensity.

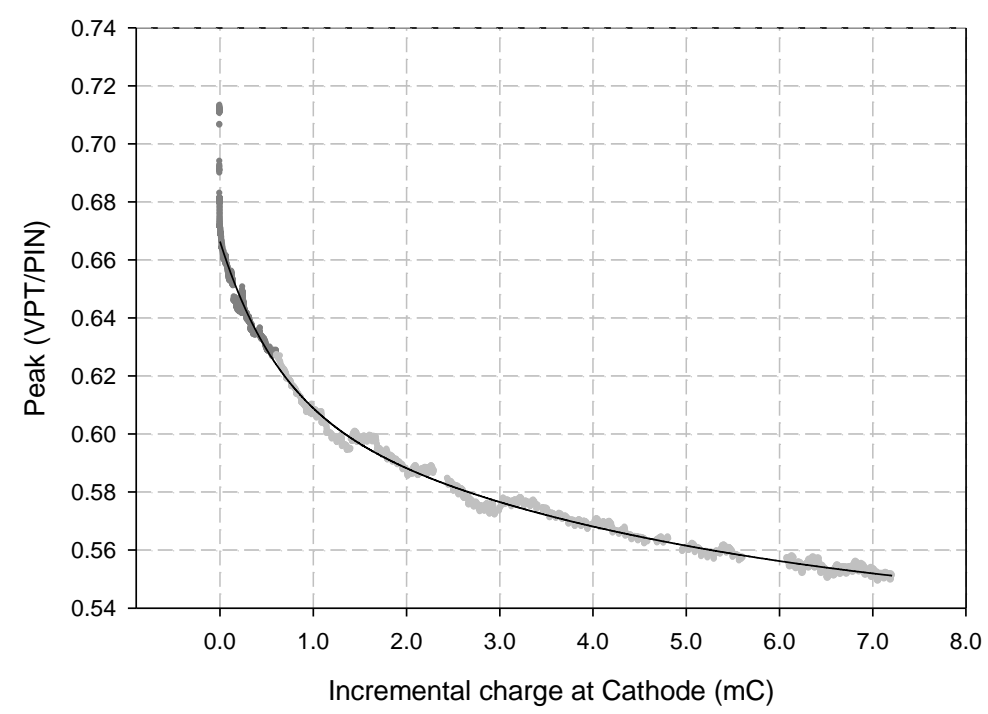

Figure 2. Dark data: load $=10 \mathrm{kHz},\left\langle\mathrm{I}_{\mathrm{C}}\right\rangle \sim 0.25 \mathrm{nA}$; Light data: $l$ load $=20 \mathrm{kHz},\left\langle\mathrm{I}_{\mathrm{C}}\right\rangle \sim 1.0 \mathrm{nA}$ 
Fitting the data shown in Figure 2 with two exponential terms (shown as a black curve in the Figure) has been found to account well for the initial fast decrease (due to 'conditioning' of the VPT) and the slower, long term decrease ('ageing'), with a constant term of 0.53 an $R^{2}$ value of 0.99 .

Figure 3 shows, in more detail, a series of seven consecutive 24-hour cycles. The Figure indicates there is a smooth downward drift, associated with 'conditioning' of the VPT and variations in response of $<0.2 \%$ which may be attributed to the simulated LHC on/off cycle. The relatively rapid, systematic changes in the VPT response, small spikes seen at 0.8 and 6.9 days (and indicated in the figure), are correlated with a small, rapid drop in the VPT preamplifier temperature when the magnet cryostat was being filled with liquid nitrogen.

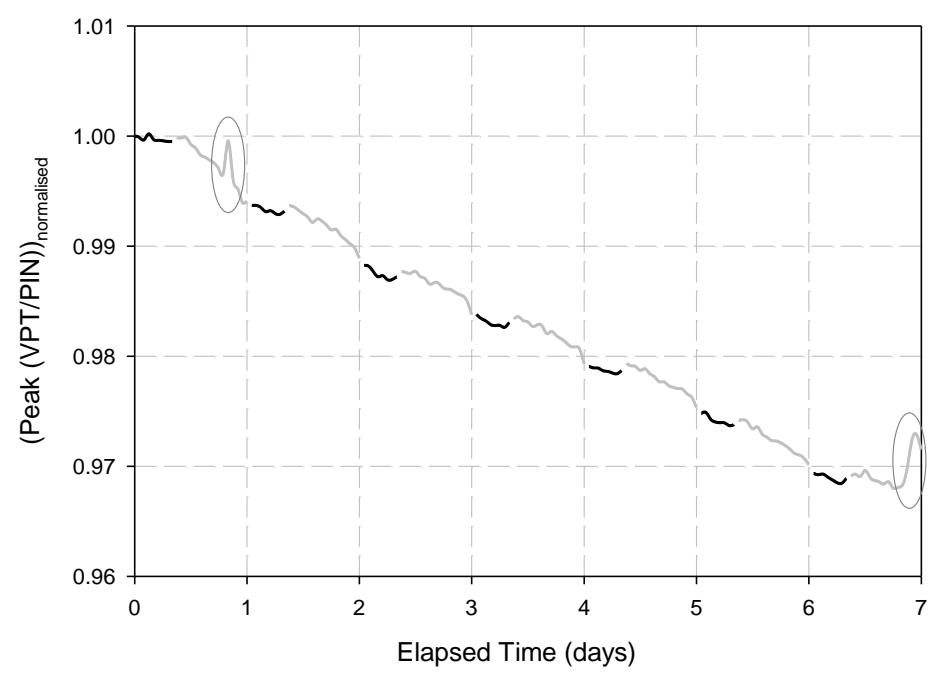

Figure 3. An example of seven consecutive cycles, where black lines indicate $100 \mathrm{~Hz}$ signal only and grey denote $100 \mathrm{~Hz}$ signal plus $20 \mathrm{kHz}$ load. In the presence of the Load pulse, $\left\langle\mathrm{I}_{\mathrm{C}}\right\rangle \sim 1.0 \mathrm{nA}$.

\section{Conclusions and Future Work}

Results of recent studies of VPT response have been discussed and in particular, the successful operation of a real VPT at full field with a realistic load for an extended period of time has been reported. From this work, a change of $<0.2 \%$ has been seen in simulated LHC on/off cycles; this meets the requirements of precision calorimetry in CMS. This extensive testing of a single VPT also 
indicates a decrease in response of $\sim 25 \%$ over six months, as expected for such vacuum photodetectors, tending towards a non-zero plateau. The studies at Brunel will continue with investigation of the effect of a lower background signal of $10 \mathrm{~Hz}$, and then will be extended to include other VPTs.

A CMS group at the University of Virginia have conducted initial tests with a 16 hour on/8 hour off cycle and an photocurrent of $10 \mathrm{nA}$, and have observed variations in the VPT response of $\sim 0.3 \%$, which have been attributed to temperature variations. Their apparatus is currently being modified to enable simultaneous testing of five VPTs at 3.8T.

It is proposed that the stability pulser on the EE at the LHC will provide the required $100 \mathrm{~Hz}$ signal and that the required short-term stability will be obtained.

\section{Acknowledgments}

We thank the technical and administrative staff at CERN and other CMS Institutes, and acknowledge support from: FMSR (Austria); FNRS and FWO (Belgium); CNPq, CAPES, FAPERJ and FAPESP (Brazil); MES (Bulgaria); CERN; CAS; MST and NSFC (China); MST (Croatia); RPF (Cyprus); Academy of Sciences and NICPB (Estonia); Academy of Finland, ME and HIP (Finland); CEA and CNRS/IN2P3 (France); BMBF, DFG and HGF (Germany); GSRT and Leventis Foundation (Greece); OTKA and NKTH (Korea); CINVESTAV, CONACYT, SEP and UASLP-FAI (Mexico); PAEC (Pakistan); SCSR (Poland); FCT (Portugal); JINR (Armenia, Belarus, Georgia, Ukraine, Uzbekistan): MST and MAE (Russia); MSD (Serbia); MCINN and CPAN (Spain); Swiss Funding Agencies (Switzerland); NSC (Taipei); TUBITAK and TAEK (Turkey); STFC (United Kingdom); DOE and NSF (USA).

\section{References}

1. The CMS Experiment at the CERN LHC, The CMS Collaboration, Chatrchyan, S et al, Journal of Instrumentation 3 (2008) S08004

2. Bell, $\mathrm{K} \mathrm{W}$ et al; Nuclear Instruments and Methods in Physics Research A 469 (2001) pp 29-46

3. National Research Institute Electron, Morisa Toreza Ave., 68, 194223 St. Petersburg, Russia

4. Performance and Operation of the CMS Crystal Electromagnetic Calorimeter, The CMS Collaboration, CMS Paper CFT-09-004 submitted to Journal of Instrumentation 